\title{
Fermi liquid instabilities in two-dimensional lattice models
}

\author{
C. A. Lamas, ${ }^{1}$ D. C. Cabra, ${ }^{1,2,3}$ and N. Grandi ${ }^{1}$ \\ ${ }^{1}$ Departamento de Física, Universidad Nacional de La Plata, Casilla de Correo 67, 1900 La Plata, Argentina \\ ${ }^{2}$ Laboratoire de Physique Théorique, Université Louis Pasteur, 3 Rue de l'Université, 67084 Strasbourg, Cédex, France \\ ${ }^{3}$ Facultad de Ingeniería, Universidad Nacional de Lomas de Zamora, Camino de Cintura y Juan XXIII, \\ 1832 Lomas de Zamora, Argentina
}

(Received 28 April 2008; revised manuscript received 25 June 2008; published 4 September 2008)

\begin{abstract}
We develop a procedure for detecting Fermi liquid instabilities by extending the analysis of Pomeranchuk to two-dimensional lattice systems. The method is very general and straightforward to apply, thus, provides a powerful tool for the search of exotic phases. We test it by applying it to a lattice electron model with interactions leading to $s$ - and $d$-wave instabilities.
\end{abstract}

DOI: 10.1103/PhysRevB.78.115104

PACS number(s): 71.10.Hf, 71.10.Fd, 71.10.Ay

\section{INTRODUCTION}

The Landau theory of the Fermi liquid (FL) is one of the most important frameworks in understanding conventional weak interacting metallic systems. ${ }^{1}$ The low-energy physics of interacting fermions in three dimensions is usually described by the Landau's FL theory, whose central assumption is the existence of single-particle fermionic excitations or "quasiparticles," with a long lifetime at very low energies. In lower dimensions, however, the situation is much more interesting; in one dimensional systems, the Landau's quasiparticles are typically unstable, giving rise to the so-called Luttinger liquid. On the other hand, two-dimensional lattice models are far more complicated to treat since conventional perturbation theory breaks down for densities close to half filling, where competing infrared divergences appear as a consequence of Fermi-surface (FS) nesting and van Hove singularities.

In Ref. 2 Pomeranchuk developed a method to diagnose instabilities of the FL by "deforming" the FS and by studying the resulting energy gain. In its original form, it applies to systems with a three-dimensional spherical FS, but it can be easily generalized to the two-dimensional continuum case.

The experimental observation of exotic phases in strongly correlated systems has triggered an enormous effort from the theoretical community to try to understand their microscopical origin. One possible route to detect instabilities of a FL is the analysis done by Pomeranchuk. Due to that, the Pomeranchuk instability has been studied by several authors with different techniques in the last few years ${ }^{3-12}$ and in particular, the instability of the FL toward the nematic phase was investigated for several models. ${ }^{13-19}$

In this paper we develop a general method to trace such instabilities in lattice models in a simple and rigorous way. It allows for the study of systems that have an arbitrary shape of the FS in the absence of interactions, thus, it becomes applicable to models relevant to high-temperature superconductors, manganites, ruthenates, etc., ${ }^{20-23}$ as long as one can rely on a perturbative analysis. It can be applied in principle to any lattice problem in a systematic way.

It should be stressed that within our method, an instability could be detected by considering infinitesimal deformations. This implies that we can detect those instabilities that lead to continuous transitions.
We test our method within two examples: the attractive Hubbard model and a model with forward-scattering interactions that give rise to $d$-wave FS deformation (the so-called " $d$-wave Pomeranchuk instability"). These examples are chosen due to their simplicity. In particular, the attractive Hubbard model provides an interesting test ground, although it does not describe the rich physics of high- $T_{c}$ superconductors.

This paper is organized as follows: Section II contains a detailed derivation of the method, with the proof of our formulas in Sec. II A, and a short-hand recipe for the application of the results in Sec. II B. Then in Sec. III we apply the method to a two-dimensional square lattice with the various interactions studied in Ref. 14, the $s$-wave interaction being studied in Sec. III B, while the $d$-wave instability in Sec. III C. Finally, Sec. IV contains the conclusions and some specific calculations are presented in the Appendix.

\section{TWO-DIMENSIONAL POMERANCHUK INSTABILITY}

\section{A. Derivation of the method}

In the theory of the Landau's FL, the free dynamics at zero temperature is determined by the dispersion relation $\varepsilon(k)$. In terms of it, the FS is defined as the set of points in momentum space satisfying the equation

$$
\mu-\varepsilon(k)=0 .
$$

In the ground state of the system, all single-particle states inside the FS $\mu-\varepsilon(k)>0$ are occupied, while those outside the FS $\mu-\varepsilon(k)<0$ are not. Excited states of the system are built by moving some particles from the inner single-particle states to the outer ones.

The energy of such excited state as a functional of the change in the equilibrium distribution function can be written as

$$
\begin{aligned}
E= & \int d^{2} k(\varepsilon(k)-\mu) \delta n(k) \\
& +\frac{1}{2} \int d^{2} k \int d^{2} k^{\prime} f\left(k, k^{\prime}\right) \delta n(k) \delta n\left(k^{\prime}\right),
\end{aligned}
$$

where $\delta n(k)$ is the change in the distribution function $n(k)$ 
and we have assumed that only two-particle interactions are present. The interaction function $f\left(k, k^{\prime}\right)$ can be related to the low-energy limit of the two-particle vertex.

Pomeranchuk criterion allows one to identify low-energy excited states of the system that make Eq. (2.2) negative. This signals an instability and the breakdown of the present FL description. In what follows, we will carefully go through all the steps needed to perform such analysis.

First let us define (associated with any given state of the system) a smooth function $g(k)$ such that it takes positive values at occupied single-particle states and negative values at unoccupied ones. Then at the frontier between these two regions, we have the equation

$$
g(k)=0 .
$$

For the ground state, such frontier coincides with the FS allowing us to choose,

$$
g(k)=\mu-\varepsilon(k) .
$$

Under a variation $\delta n(k)$ of the distribution function $n(k)$, we get an excited state that can be described in terms of a new function $g^{\prime}(k)=g(k)+\delta g(k)$. The frontier between occupied and unoccupied single-particle states is now located at points satisfying

$$
g^{\prime}(k)=g(k)+\delta g(k)=0 .
$$

By an abuse of language we will call the solution of this equation the deformed $F S$.

Since at $T=0$ we have $\delta n(k)= \pm 1$, we can write

$$
\delta n(k)=H\left[g^{\prime}(k)\right]-H[g(k)],
$$

where $H(x)$ is the unit step function defined by $H(x)=1$ if $x>0$ and $H(x)=0$ if $x<0$. This can be replaced in Eq. (2.2) to write the energy of the quasiparticles as a functional of $g(k)$ and $g^{\prime}(k)$, namely,

$$
\begin{aligned}
E= & \int d^{2} k(\varepsilon(k)-\mu)\left(H\left[g^{\prime}(k)\right]-H[g(k)]\right) \\
& +\frac{1}{2} \int d^{2} k \int d^{2} k^{\prime} f\left(k, k^{\prime}\right)\left(H\left[g^{\prime}(k)\right]-H[g(k)]\right)\left(H\left[g^{\prime}\left(k^{\prime}\right)\right]\right. \\
& \left.-H\left[g\left(k^{\prime}\right)\right]\right) .
\end{aligned}
$$

To go further, we have to take into account the constraint imposed by the Luttinger theorem, ${ }^{24}$ or in other words, the preservation of the area of the FS under the deformation,

$$
\int d^{2} k \delta n(k) \equiv 0
$$

By using Eq. (2.6) this can be rewritten as a functional constraint on the functions $g(k)$ and $g^{\prime}(k)$,

$$
\int d^{2} k H\left[g^{\prime}(k)\right]=\int d^{2} k H[g(k)] .
$$

In two dimensions the constraint (2.9) can be easily solved as follows. We first rename integration variables on the right-hand side to $k^{\prime}$. Next we assume that a change of variables $k^{\prime}=k+\delta k(k)$ can take the right-hand side into the form of the left-hand side. Writing $g^{\prime}(k)=g(k)+\delta g(k)$, we get two unknown functions, namely, $\delta g(k)$ and $\delta k(k)$, together with the equation

$$
\int d^{2} k H[g(k)+\delta g(k)]=\int d^{2} k\left|1+\partial_{j} \delta k^{i}\right| H[g(k+\delta k(k))],
$$

where $i, j \in\{1,2\}$ label the orthogonal directions in momentum space.

A particular class of solutions can then be obtained by solving the following equations:

$$
\begin{gathered}
\left|1+\partial_{j} \delta k^{i}\right|=1, \\
g(k)+\delta g(k)=g(k+\delta k(k)) .
\end{gathered}
$$

The first line of Eq. (2.11) implies that the change of variables going from $k^{\prime}$ to $k$ is an area preserving diffeomorphism. The second line, on the other hand, can be interpreted as saying that the variation $\delta g(k)$ is a translation of $g(k)$ by an amount of $\delta k$. To solve this equation one uses the identity $\operatorname{det}(1+A)=\exp (\operatorname{tr}[\log (1+A)])$ and expands the right-hand side of this equation up to second order in $A$. Since the determinant is quadratic in $A$, the resulting expression is exact. It can then be solved to linear order by

$$
\delta k^{i}=\epsilon^{j k} \partial_{j} \lambda
$$

and then an iterative procedure can be used to get the full solution,

$$
\begin{gathered}
\delta k^{i}=\left(e^{\varepsilon^{j k} \partial_{j} \lambda \partial_{k}}-1\right) k^{i}, \\
\delta g=\left(e^{\epsilon^{i j} \partial_{i} \lambda \partial_{j}}-1\right) g .
\end{gathered}
$$

Where $\lambda$ is a free function parameterizing the deformation. If we assume that the deformation of the FS is small, then $\delta g(k)$ is also small and we can parameterize it in terms of a slowly varying $\lambda$,

$$
\begin{gathered}
\delta k^{i} \simeq \epsilon^{i j} \partial_{j} \lambda, \\
\delta g \simeq \epsilon^{i j} \partial_{j} \lambda \partial_{i} g .
\end{gathered}
$$

In what follows, each specific form of $\lambda$ will characterize an excited state and the sign of the resulting energy will give us information about the instabilities.

Other solutions that could in turn lead to a larger instability region may exist. In other words, our method is well suited to detect instabilities but not to diagnose stability.

Now that we have solved the constraint, we go back to the energy of the quasiparticles (2.7) and write it in terms of the free unconstrained variable $\lambda$. To simplify the resulting expression, we need to change variables to a more convenient coordinate system in momentum space. We choose a special set of variables,

$$
\begin{gathered}
g=g\left(k_{x}, k_{y}\right), \\
s=s\left(k_{x}, k_{y}\right),
\end{gathered}
$$

where the new variable $g$ varies in the direction transverse to the unperturbed FS. The variable $s$ varies in the longitudinal 
direction tangent to the FS, namely, it satisfies $\partial_{i} s \partial_{i} g=0$.

Separating the energy (2.7) into a linear term and an interaction term $E=L+I$, we get for the linear part,

$$
\begin{aligned}
L & =\int d^{2} k(\varepsilon(k)-\mu)(H[g+\delta g]-H[g]) \\
& =\int d s d g J(s, g)(\varepsilon(s, g)-\mu)(H[g+\delta g]-H[g]) \\
& =\int d s \int_{-\delta g}^{0} d g J(s, g)(\varepsilon(s, g)-\mu),
\end{aligned}
$$

where $J=\left|\partial\left(k_{x}, k_{y}\right) / \partial(g, s)\right|$ is the Jacobian of the transformation (2.14). Expanding in powers of the integration variable $g$ around the unperturbed FS $g=0$, we get

$$
\begin{aligned}
L & =\int d s \int_{-\delta g}^{0} d g \partial_{\bar{g}}[J(s, \bar{g})(\varepsilon(s, \bar{g})-\mu)]_{\bar{g}=0} g+\mathcal{O}\left(\delta g^{2}\right) \\
& =\frac{1}{2} \int d s\left[J(s, \bar{g}) \delta g^{2}\right]_{g=g^{\prime}=0}+\mathcal{O}\left(\delta g^{3}\right)
\end{aligned}
$$

where in the second line we have integrated out the variable $g$ and made use of the fact that $(\varepsilon(s, g=0)-\mu)=0$. In order to replace the explicit form of $\delta g$ [Eq. (2.13)] in the integrand of Eq. (2.16), we make use of the identity

$$
\begin{aligned}
\epsilon^{i j} \partial_{i} g \partial_{j} \lambda & =\epsilon^{i j}\left(\partial_{i} g \partial_{g} g+\partial_{i} s \partial_{s} g\right)\left(\partial_{j} g \partial_{g} \lambda+\partial_{j} s \partial_{s} \lambda\right) \\
& =\left(\epsilon^{i j} \partial_{i} g \partial_{j} s\right) \partial_{s} \lambda \equiv J^{-1} \partial_{s} \lambda,
\end{aligned}
$$

where we have used the fact that (according to our definitions) $\partial_{g} g=1$ and $\partial_{s} g=0$, the antisymmetry of the $\epsilon^{i j}$ tensor, and the definition of the determinant. Now replacing in Eq. (2.16) we get

$$
L=\frac{1}{2} \int d s\left[J^{-1}(g, s)\left(\partial_{s} \lambda\right)^{2}\right]_{g=0} .
$$

The calculus of $I$ is analogous and gives

$$
I=\left.\frac{1}{2} \int d s \int d s^{\prime}\left[f\left(g, s ; g^{\prime}, s^{\prime}\right)\left(\partial_{s} \lambda\right)\left(\partial_{s^{\prime}} \lambda\right)\right]\right|_{g=g^{\prime}=0} .
$$

Adding the two contributions we finally have

$$
\begin{aligned}
E= & \frac{1}{2} \int d s \int d s^{\prime}\left(f\left(0, s ; 0, s^{\prime}\right)+J^{-1}(0, s) \delta\left(s-s^{\prime}\right)\right) \\
& \times \partial_{s} \lambda(0, s) \partial_{s^{\prime}} \lambda\left(0, s^{\prime}\right) .
\end{aligned}
$$

As the functions $\lambda(0, s)$ characterizing the excited states are arbitrary, we can equally work with functions $\psi(s)$ $=\left.\partial_{s} \lambda(g, s)\right|_{g=0}$. In what follows we will be interested in excited states such that $\psi(s) \in L_{2}[0, S]$. Assuming that $s$ makes a complete turn around the FS when it runs from 0 to $S$, we also need to impose periodicity in that interval.

Since the sign of $E$ in Eq. (2.20) determines the stability of the FL, from all the above we conclude that the stability condition reads

$$
E=\int d s^{\prime} \int d s \psi\left(s^{\prime}\right) \frac{1}{2}\left(J^{-1}(s) \delta\left(s-s^{\prime}\right)+f\left(s, s^{\prime}\right)\right) \psi(s)>0,
$$

where have we defined

$$
\begin{gathered}
f\left(s, s^{\prime}\right)=\left.f\left(g, s ; g^{\prime}, s^{\prime}\right)\right|_{g=g^{\prime}=0}, \\
J^{-1}(s)=\left.J^{-1}(g, s)\right|_{g=0} .
\end{gathered}
$$

Note that the stability condition has two terms; the first of which contains the information about the form of the FS via $J^{-1}(s)$, while the second encodes the specific form of the interaction in $f\left(s, s^{\prime}\right)$. There is a clear competition between the interaction function in the second term of the integrand and the first term that only depends on the geometry of the unperturbed FS.

We see that $E$ is a bilinear form, acting on the real functions $\psi(s)$ parameterizing the deformations of the FS,

$$
E=\langle\psi, \psi\rangle,
$$

where

$$
\langle u, v\rangle=\int d s^{\prime} \int d s u(s) \frac{1}{2}\left(f\left(s, s^{\prime}\right)+J^{-1}(s) \delta\left(s-s^{\prime}\right)\right) v\left(s^{\prime}\right) .
$$

The stability condition is then equivalent to asking this form to be positive definite for any possible deformation, i.e.,

$$
\forall \psi:\langle\psi, \psi\rangle>0
$$

In consequence, the natural way to diagnose an instability is to diagonalize this bilinear form and to look for negative eigenvalues.

We can expand the functions $\psi(s)$ in some basis of $L^{2}[0, S]$ that we will denote $\left\{\xi_{i}(s)\right\}$,

$$
\psi(s)=\sum_{i} a_{i} \xi_{i}(s),
$$

and then write

$$
E=\sum_{i_{1}, i_{2}} a_{i_{1}} a_{i_{2}}\left\langle\xi_{i_{1}}, \xi_{i_{2}}\right\rangle .
$$

The bilinear form $\langle$,$\rangle can be taken as a pseudoscalar prod-$ uct, which is linear and symmetric but, in general, not positive definite. Only in the free case $f\left(s, s^{\prime}\right) \equiv 0$ the positivity is ensured. If $\left\{\xi_{i}(s)\right\}$ are taken to be orthogonal with respect to this pseudoscalar product, then the functional (2.21) is given by

$$
E=\sum_{i} a_{i}^{2} \chi_{i}^{\mu},
$$

where $\chi_{i}^{\mu}=\left\langle\xi_{i}, \xi_{i}\right\rangle$ is the square pseudonorm of the orthogonal functions. If $\chi_{i}^{\mu}$ has a negative value for some $i$, then by choosing the corresponding $a_{i}^{2}=1$ and $a_{j}^{2}=0$ for $j \neq i$, we see that the energy is negative denoting an instability. In this case we say that we have an instability in the $i$ th channel. In other words, the stability condition has been mapped into 


$$
\forall i: \chi_{i}^{\mu}>0,
$$

with $\chi_{i}^{\mu}$ being taken as the stability parameters. If any of these quantities is negative, then the FS is unstable.

We perform such diagonalization by choosing a basis on $L_{2}[0, S]$ as a given set of functions $\left\{\psi_{i}\right\}$ and then making use of the Gram-Schmidt orthogonalization procedure to transform it into an orthogonal basis $\left\{\xi_{i}\right\}$. Note that, the bilinear form being not necessarily positive definite, the new basis cannot be normalized to 1 but to \pm 1 .

This is our main result. Our method to search for Pomeranchuk instabilities can be summarized in the recipe presented in Sec. II B.

\section{B. Recipe}

(i) Get the dispersion relation $\varepsilon(k)$ and the interaction function $f\left(k, k^{\prime}\right)$ for the model under study.

(ii) Change the variables according to Eq. (2.14). The variable $g$ is completely fixed by the dispersion relation according to Eq. (2.4). The choice of $s$ is arbitrary except for the constraint of being tangential to the FS $\left(\partial_{i} s \partial_{i} g=0\right) .{ }^{25}$

(iii) Write the bilinear form $E$ as in Eq. (2.21).

(iv) Choose an arbitrary basis of functions $\left\{\psi_{i}\right\}$ of $L_{2}[0, S]$.

(v) Apply the Gram-Schmidt orthogonalization procedure, verifying at each step whether $\left\langle\xi_{i}, \xi_{i}\right\rangle>0$.

(vi) If for a given channel $i$ one finds that $\left\langle\xi_{i}, \xi_{i}\right\rangle<0$, the FS is diagnosed to be unstable.

Note that since $L_{2}[0, S]$ is infinite dimensional, the present method is not efficient to verify stability; at any step $i$, it may always be the case that for some $j, \chi_{i+j}^{\mu}<0$. Moreover, we have not exhausted all the possible solutions of the constraint (2.9) but only explored a subset of them.

\section{INSTABILITIES IN THE SQUARE LATTICE}

\section{A. Contribution of the free Hamiltonian}

We start considering free fermions in the square lattice with a Hamiltonian given by

$$
H_{0}=\sum(\varepsilon(k)-\mu) c_{k}^{\dagger} c_{k},
$$

where

$$
\varepsilon(k)=-2 t\left(\cos k_{x}+\cos k_{y}\right),
$$

where only hopping to nearest neighbors has been taken into account. The FS is defined by

$$
g(k)=\mu-\varepsilon(k)=\mu+2 t\left(\cos k_{x}+\cos k_{y}\right)=0,
$$

where $\mu$ is the chemical potential. Notice that $g>0$ inside the area bounded by the FS, negative outside it, and zero at the FS.

Now we follow the recipe given in Sec. II B, changing variables according to Eq. (2.14),

$$
g\left(k_{x}, k_{y}\right)=\mu+2 t\left(\cos k_{x}+\cos k_{y}\right)
$$

$$
s\left(k_{x}, k_{y}\right)=\arctan \left(\frac{\tan \left(k_{y} / 2\right)}{\tan \left(k_{x} / 2\right)}\right) .
$$

It is straightforward to see that $g$ and $s$ are mutually orthogonal variables. Using the following shorthand notation:

$$
\begin{aligned}
& \alpha=\cos k_{x}, \\
& \beta=\cos k_{y},
\end{aligned}
$$

we can write

$$
\begin{gathered}
g=\mu+2 t(\alpha+\beta), \\
\tan ^{2}(s)=\left(\frac{1-\beta}{1+\beta}\right)\left(\frac{1+\alpha}{1-\alpha}\right),
\end{gathered}
$$

and the Jacobian takes the form

$$
J=t\left(\frac{\alpha \beta-1}{\alpha^{2}+\beta^{2}-2}\right) .
$$

Notice that $J \geq 0$. Writing $\alpha$ and $\beta$ as functions of $g$ and $s$ we have for the Jacobian evaluated at $g=0$,

$$
J[g=0, s]=\frac{1}{2 t \sqrt{1-\beta(\mu) \cos ^{2}(2 s)}},
$$

with $\beta(\mu)=1-\left(\frac{\mu}{4 t}\right)^{2}$. The limits for the variable $s$ can be taken as $-\pi<s \leq \pi$.

The inverse of the Jacobian $J^{-1}(s)$ can be expanded in series of $\sin (n s)$ and $\cos (n s)$ and it is straightforward to show that only the coefficients of $\cos (4 n s)$ are nonvanishing. This results in the following expansion:

$$
J^{-1}(s)=\sum_{n} j_{n}^{\mu} \cos (4 n s),
$$

where the coefficients $j_{n}^{\mu}$ are fixed by the expansion. Some of them are given in the Appendix. The simplicity of this expansion suggests to take the set $\{\sin (n s), \cos (n s)\}$ as our starting base in the Gram-Schmidt orthogonalization procedure.

In Secs. III B and III C we will analyze, as an example of application, the possible instabilities in this two-dimensional fermion model when subjected to various interactions. In particular we are interested in interactions of the form, ${ }^{14}$

$$
f\left(k, k^{\prime}\right)=(\text { Constant })\left[d(k) d\left(k^{\prime}\right)\right],
$$

with

$$
\begin{gathered}
d(k)=1, \quad(s \text {-wave }), \\
d(k)=\left(\cos k_{x}+\cos k_{y}\right), \quad(\text { extended } s \text {-wave }), \\
d(k)=\left(\cos k_{x}-\cos k_{y}\right), \quad\left(d \text {-wave } d_{x^{2}-y^{2}}\right)
\end{gathered}
$$

\section{B. $s$-wave Pomeranchuk instability}

We first consider a constant interaction corresponding to take $^{14} d(k)=1$ so that 

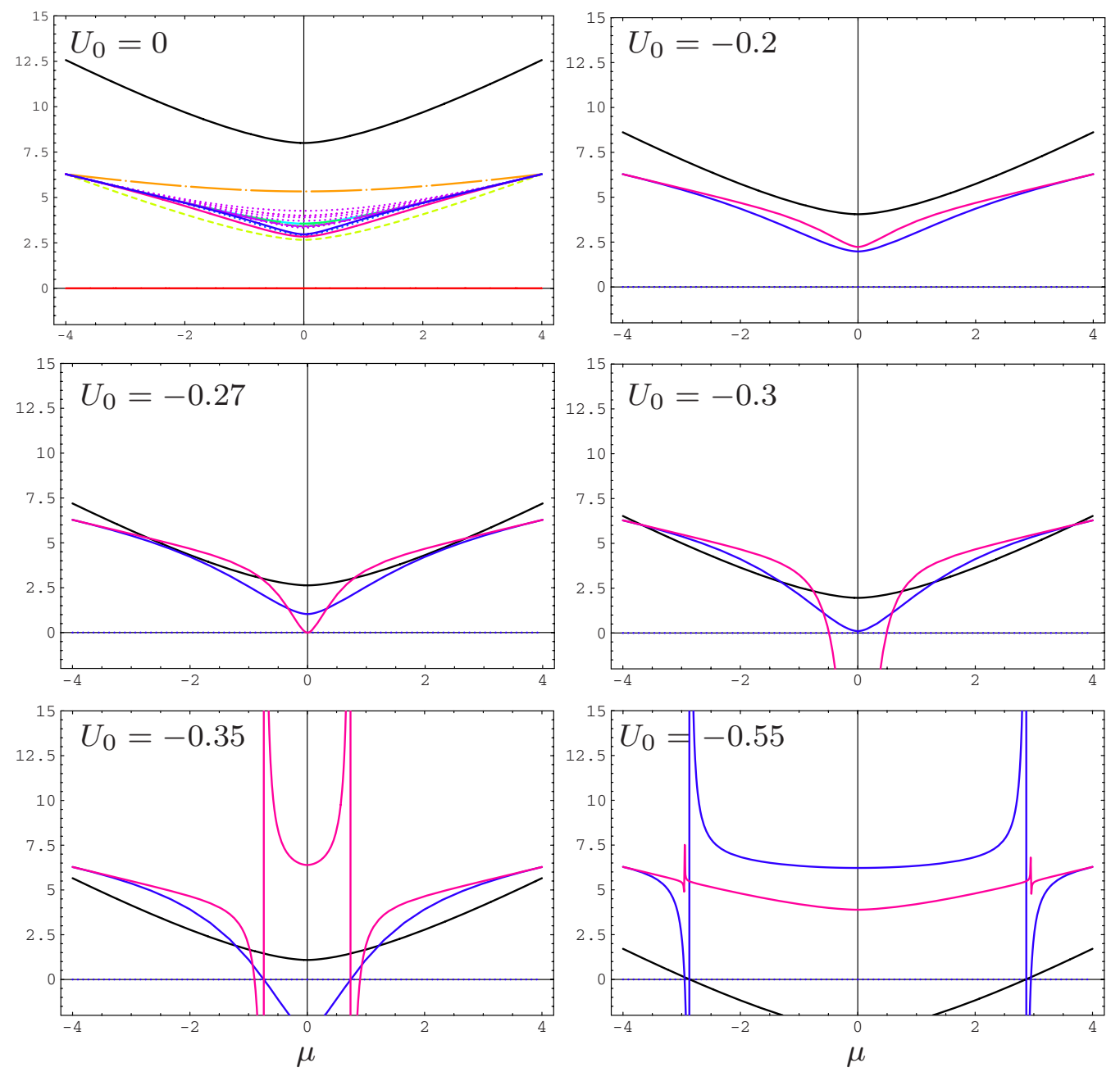

FIG. 1. (Color online) The instability parameters. For $U_{0}=0$ we show the first ten parameters $\chi_{i}^{\mu}$ as a function of $\mu$. For other values of the interaction, we show only the parameters corresponding to the three lower channels that show instabilities, namely, channels $\chi_{0}, \chi_{8}$, and $\chi_{16}$ (colors are identified in Fig. 2). Notice that when we increase $U_{0}$, the breakdown of the FL description under consideration occurs first for the higher channels and closer to half filling.

$$
f\left(k, k^{\prime}\right)=U_{0},
$$

with $U_{0}$ being a constant measuring the strength of the interaction. This form of the interaction can be obtained by a mean-field approximation or a first-order perturbative expansion for the interaction function on the Hubbard model ${ }^{26,27}$ It should be noticed that this interaction preserves the full symmetry of the dispersion relation.

Using the product defined in Eq. (2.24) we can calculate the first instability parameters.

$$
\begin{gathered}
\chi_{0}^{\mu}=2 \pi\left(U_{0} \pi+j_{0}^{\mu}\right), \\
\chi_{1,2}^{\mu}=\pi j_{0}^{\mu}, \\
\chi_{3}^{\mu}=\pi\left(j_{0}^{\mu}-\frac{1}{2} j_{1}^{\mu}\right), \\
\chi_{4}^{\mu}=\pi\left(j_{0}^{\mu}+\frac{1}{2} j_{1}^{\mu}\right),
\end{gathered}
$$

$$
\begin{gathered}
\chi_{5,6}^{\mu}=-\frac{\pi}{2} \frac{j_{1}^{\mu 2}}{j_{0}^{\mu}}+j_{0}^{\mu}\left(\pi+\frac{\pi}{4}\left(\frac{j_{1}^{\mu}}{j_{0}^{\mu}}\right)^{2}\right), \\
\chi_{7}^{\mu}=\pi\left(j_{0}^{\mu}-\frac{1}{2} j_{2}^{\mu}\right), \\
\chi_{8}^{\mu}=\frac{\pi^{2} U_{0} j_{1}^{\mu 2}}{2\left(\pi U_{0}+j_{0}^{\mu}\right)^{2}}-\frac{\pi j_{1}^{\mu 2}}{\pi U_{0}+j_{0}^{\mu}} \\
+j_{0}^{\mu}\left(\pi+\frac{\pi j_{1}^{\mu 2}}{2\left(\pi U_{0}+j_{0}^{\mu}\right)^{2}}\right)+\frac{\pi j_{2}^{\mu}}{2},
\end{gathered}
$$

The stability parameters for the first channels are shown in Fig. 1. For $U_{0}=0$ all the $\chi_{n}^{\mu}$ are positive as expected. When we increase the interaction, among the first 20 parameters, only $\chi_{0}^{\mu}, \chi_{8}^{\mu}$, and $\chi_{16}^{\mu}$ change. For simplicity only these parameters are plotted for $U_{0} \neq 0$. 


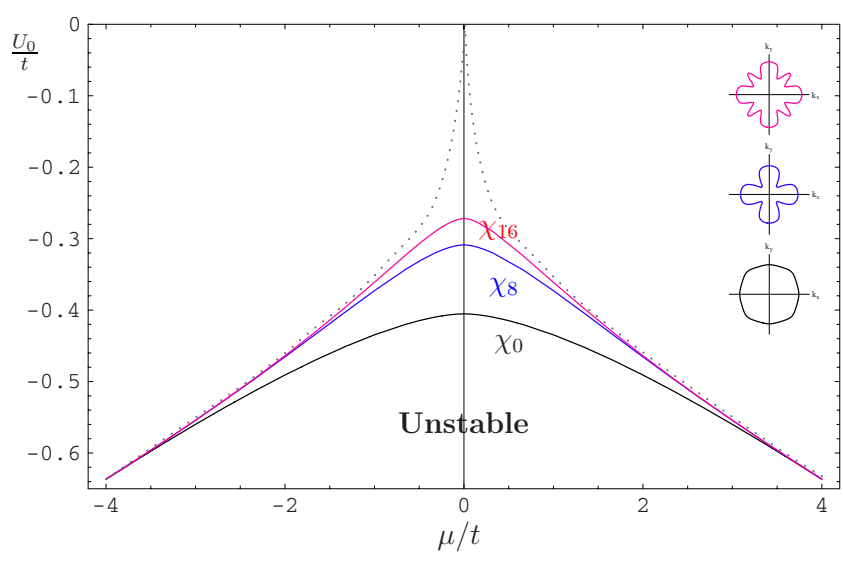

FIG. 2. (Color online) Phase diagram for $f\left(s, s^{\prime}\right)=U_{0}$. The regions of instability for the first three unstable channels are displayed. For smaller interactions, higher channels are unstable closer to half filling and extrapolate to the dotted curve. The shape of the unstable FS deformations are displayed and one can observe that only modes that preserve the symmetry of the total energy show instabilities.

A sketch of the deformed Fermi surface (FS) for the first three unstable channels is shown in Fig. 2. As it might be expected, the channels showing instability are those that preserve the symmetry of the total energy, which in the present case corresponds to the symmetry of the dispersion relation.

With these first instability channels we can draw a qualitative phase diagram in the $\left(\mu, U_{0}\right)$ space as in Fig. 2, where the first instability zones are shown and a tentative global phase diagram is drawn.

Note that, when the interaction is increased, the first instability channel corresponds to the highest of the three shown in the figure (e.g., $\chi_{16}^{\mu}$ ). This behavior is maintained for channels $\chi_{i}^{\mu}$ with higher index $i$, and we assume that generically these higher channels will show the instability closer to half filling and for interactions arbitrarily small. On the other hand, the higher the channel, the closer the instability region is to half filling. Extrapolating this behavior, we see that the instabilities on the large- $i$ channels take place only very close to $\mu=0$.

The behavior for the extended $s$ wave with a form factor $d(k)=\left(\cos k_{x}+\cos k_{y}\right)=\alpha+\beta$ can be studied by writing $d(k)$ in terms of the variables $g$ and $s$ using the solutions of Eq. (3.8) and by evaluating at $g=0$. We have $d(s)=\frac{-\mu}{2 t}$ and the interaction function reads

$$
f\left(s, s^{\prime}\right)=U_{0}\left(\frac{\mu}{2 t}\right)^{2} .
$$

Again $f\left(s, s^{\prime}\right)$ is independent of the variables $s$ and $s^{\prime}$ but now dependent of the chemical potential $\mu$.

The corresponding instability parameters are obtained by changing $U_{0} \rightarrow U_{0}\left(\frac{\mu}{2 t}\right)^{2}$ in Eq. (3.15) and the phase diagram can then be inferred to be analogous to that of Fig. 2 but with the vertical axes replaced by $U_{0}\left(\frac{\mu}{2 t}\right)^{2}$.

\section{C. $d$-wave Pomeranchuk instability}

Now we investigate the $d$-wave Fermi-surface deformation (dFSD) instability ${ }^{28}$ in the charge channel on a square lattice. The forward-scattering interaction driving the dFSD has the form ${ }^{14}$

$$
f\left(k, k^{\prime}\right)=-G d(k) d\left(k^{\prime}\right),
$$

with $G>0$ and $d$-wave form factors $d(k)=\left(\cos k_{x}-\cos k_{y}\right)$. The above expression for this effective interaction was obtained by Metzner et al. ${ }^{14}$ using functional renormalizationgroup methods.

Notice that this interaction has a lower symmetry than the dispersion relation. We hence expect that deformations leading to instabilities will break the symmetry of the FS while preserving this lower one.

Using the short-hand notation (3.6) the interaction reads

$$
f\left(s, s^{\prime}\right)=-G(\alpha-\beta)\left(\alpha^{\prime}-\beta^{\prime}\right)
$$

and using the solution of Eq. (3.8) we have

$$
d(s)=\frac{2}{\cos (2 s)}\left(\frac{J^{-1}(s)}{2}-1\right) .
$$

Notice that this interaction contains the Jacobian but its origin is totally independent of the treatment developed in Sec. III B.

The form factor $d(s)$ can be expanded in a series of the form

$$
d(s)=\sum_{k=0}^{\infty} d_{k} \cos [(4 k+2) s]
$$

where the first coefficients of the expansion are presented in the Appendix.

Performing the Gram-Schmidt orthogonalization as in previous case, we find the $\chi^{\mu}$ parameters corresponding to this interaction. The results are very similar and we will only display here the first two parameters that show an instability of the system, namely,

$$
\begin{aligned}
\chi_{4}^{\mu}=-2 G \pi^{2} d_{0}^{2}+\pi j_{0}+\frac{\pi j_{1}}{2} \\
\chi_{12}^{\mu}=\frac{\pi}{2 g \pi 4 d_{0}^{2}-2\left(2 j_{0}+j_{1}\right)}\left(j_{1}^{2}-4 j_{0}^{2}+2 j_{1} j_{2}+j_{2}^{2}-j_{1} j_{3}\right. \\
-2 j_{0}\left(j_{1}+j_{3}\right)+g \pi\left(2 d_{1}^{0}\left(2 j_{0}+j_{1}\right)-8 d_{0} d_{1}\left(j_{1}+j_{2}\right)\right. \\
\left.\left.+4 d_{0}^{2}\left(2 j_{0}+j_{3}\right)\right)\right)
\end{aligned}
$$

Again, by making use of this first instabilities we can sketch the phase diagram corresponding to this interaction as shown in Fig. 3. Similar to the previous case, as the interaction grows, instabilities appear first in the higher channels. The dashed line corresponds to the critical value of the interaction found in Ref. 15 by means of a mean-field procedure. Notice that the lowest channels cover most of the instability zone. The phase diagram shown in Fig. 3 is consistent with the results presented in Refs. 15 and 18.

As advanced, deformations of the FS leading to instabilities break the symmetry of the original FS down to the symmetry of the total energy.

While our method detects continuous transitions, a firstorder one with a $d$-wave FS deformation was found by 


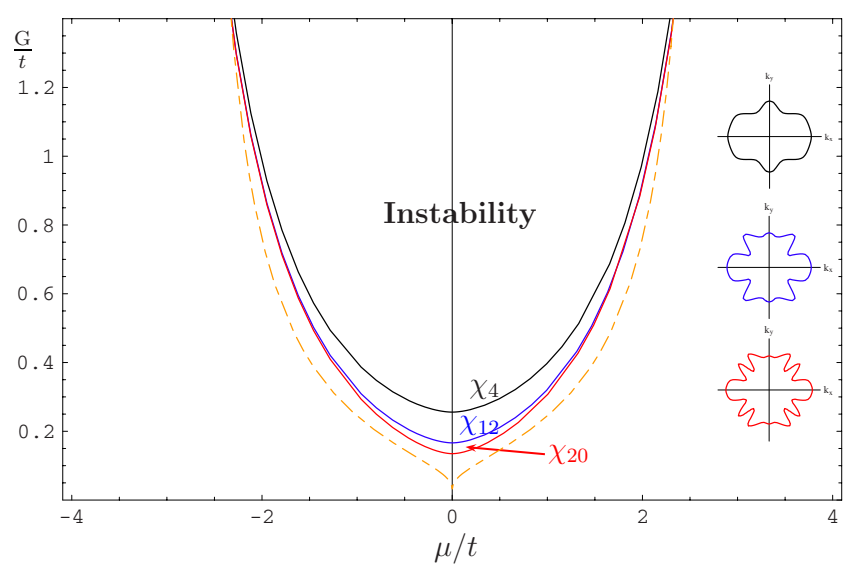

FIG. 3. (Color online) Phase diagram for the $d$-wave interaction. The first three unstable channels are shown together with the corresponding deformed FSs. The dashed line corresponds to the value for the interaction parameter studied in Ref. 15 within a mean-field treatment. Again, one observes that the symmetry of the deformations are those of the total energy.

mean-field treatments in Refs. 15 and 18. Indeed, we cannot ensure that the FL is stable outside the instability region we found, for example, with respect to discontinuous transitions. This implies that the true instability region could be larger than the one found with our method.

Unlike treatments using mean field, with the present formalism, it is possible to identify the region in the parameter space where each channel presents a breakdown of the considered FL description.

\section{SUMMARY AND CONCLUSIONS}

In this paper we have developed a general procedure for detecting instabilities in two-dimensional lattice models. It is an extension of the formalism of Landau-Pomeranchuk, in particular, for lattice systems with an arbitrary-shaped FS and allows one to describe the phase diagram of the system as an alternative to the usual procedures. The steps are simple and applicable to a wide variety of systems.

Complementary to other descriptions, our procedure permits one to identify the instability on each channel independently.

As a form of testing our procedure, we have analyzed the stability of the FL in a square lattice for various interactions already studied in the literature. The $s$-wave instability in the electronic channel and the instability produced by $d$-wave forward-scattering interactions were studied at $T=0$. The instabilities corresponding to low channels produce a breakdown of the considered FL behavior for a wide range of fillings, while those occurring for higher channels are closer to half filling. Our results are in good agreement with those obtained by different methods that were previously published by other authors.

From the results obtained one observes that, generically, instability channels preserve the symmetry of the total energy. Then, to increase the efficiency of the method, one could order the initial basis starting with those functions which preserve such symmetry.
Generalization to higher dimensions, spin-dependent interactions, or finite temperature can be achieved by following the same lines and the results will be presented elsewhere..$^{29}$

\section{ACKNOWLEDGMENTS}

We would like to thank E. Fradkin for helpful discussions. This work was partially supported by the ESF grant INSTANS, ECOS-Sud Argentina-France collaboration (Grant No. A04E03), PICS CNRS-Conicet (Grant No. 18294), PICT ANCYPT (Grant No. 20350), and PIP CONICET (Grant No. 5037).

\section{APPENDIX: ORTHOGONAL BASIS AND SERIES EXPANSION}

In this Appendix we present the coefficients in the expansion of the functions used in this paper. For the Jacobian, the series takes the form

$$
J^{-1}(s)=\sum_{n} j_{n}^{\mu} \cos (4 n s),
$$

where the first coefficients in the expansion are given by

$$
\begin{gathered}
j_{0}^{\mu}=\frac{4}{\pi} \mathbf{E}\left[1-\frac{\mu^{2}}{16}\right], \\
j_{1}^{\mu}=\frac{1}{\pi}\left(|\mu| \mathbf{E}\left[1-\frac{16}{\mu^{2}}\right]-4 \mathbf{E}\left[1-\frac{\mu^{2}}{16}\right]\right. \\
-|\mu| \frac{\pi}{2}{ }_{2} \mathbf{F}_{\mathbf{1}}\left[-\frac{1}{2}, \frac{3}{2}, 2,1-\frac{16}{\mu^{2}}\right] \\
\left.+2 \pi_{2} \mathbf{F}_{1}\left[-\frac{1}{2}, \frac{3}{2}, 2,1-\frac{\mu^{2}}{16}\right]\right), \\
j_{2}^{\mu}=\frac{4}{\pi}\left(2 \mathbf{E}\left[1-\frac{\mu^{2}}{16}\right]-4 \pi_{2} \mathbf{F}_{1}\left[-\frac{1}{2}, \frac{3}{2}, 2,1-\frac{\mu^{2}}{16}\right]\right. \\
\left.+3 \pi_{2} \mathbf{F}_{1}\left[-\frac{1}{2}, \frac{5}{2}, 3,1-\frac{\mu^{2}}{16}\right]\right), \\
j_{3}^{\mu}=\frac{-8}{\pi}\left(\mathbf{E}\left[1-\frac{\mu^{2}}{16}\right]+4 \pi\left(9_{2} \mathbf{F}_{1}\left[-\frac{1}{2}, \frac{3}{2}, 2,1-\frac{\mu^{2}}{16}\right]\right.\right. \\
+18_{2} \mathbf{F}_{1}\left[-\frac{1}{2}, \frac{5}{2}, 3,1-\frac{\mu^{2}}{16}\right] \\
\left.\left.+10_{2} \mathbf{F}_{1}\left[-\frac{1}{2}, \frac{7}{2}, 4,1-\frac{\mu^{2}}{16}\right]\right)\right),
\end{gathered}
$$

where $\mathbf{E}[m]$ is the complete elliptic integral 


$$
\mathbf{E}[m]=\int_{0}^{\pi / 2} \sqrt{1-m \sin ^{2}(t)} d t
$$

and ${ }_{2} \mathbf{F}_{\mathbf{1}}(a, b ; c ; z)$ is the hypergeometric function

$$
{ }_{2} \mathbf{F}_{1}(a, b ; c ; z)=\frac{\Gamma(c)}{\Gamma(b) \Gamma(-b+c)} \int_{0}^{1} t^{b-1}(1-t)^{c-b-1}(1-t z)^{-a} d t .
$$

The form factor for the $d$-wave forward-scattering interaction can be expanded as follows:

$$
d(s)=\sum_{k=0}^{\infty} d_{k} \cos ((4 k+2) s),
$$

with

$$
\begin{gathered}
d_{0}^{\mu}=\frac{-4\left(\pi-2 \mathbf{E}\left[1-\frac{\mu^{2}}{16}\right]\right)}{\pi}, \\
d_{1}^{\mu}=\frac{4}{\pi}\left(\pi-6 \mathbf{E}\left[1-\frac{\mu^{2}}{16}\right]+2 \pi_{2} \mathbf{F}_{1}\left[-\frac{1}{2}, \frac{3}{2}, 2,1-\frac{\mu^{2}}{16}\right]\right),
\end{gathered}
$$

$$
\begin{aligned}
d_{2}^{\mu}= & \frac{-4}{\pi}\left(\pi-10 \mathbf{E}\left[1-\frac{\mu^{2}}{16}\right]+10 \pi_{2} \mathbf{F}_{\mathbf{1}}\left[-\frac{1}{2}, \frac{3}{2}, 2,1-\frac{\mu^{2}}{16}\right]\right. \\
& \left.-6 \pi_{2} \mathbf{F}_{\mathbf{1}}\left[-\frac{1}{2}, \frac{5}{2}, 3,1-\frac{\mu^{2}}{16}\right]\right), \\
d_{3}^{\mu}= & \frac{4}{\pi}\left(-14 \mathbf{E}\left[1-\frac{\mu^{2}}{16}\right]+\pi\left(1+28_{2} \mathbf{F}_{\mathbf{1}}\left[-\frac{1}{2}, \frac{3}{2}, 2,1-\frac{\mu^{2}}{16}\right]\right.\right. \\
& -42_{2} \mathbf{F}_{\mathbf{1}}\left[-\frac{1}{2}, \frac{5}{2}, 3,1-\frac{\mu^{2}}{16}\right] \\
& \left.\left.+20_{2} \mathbf{F}_{\mathbf{1}}\left[-\frac{1}{2}, \frac{7}{2}, 4,1-\frac{\mu^{2}}{16}\right]\right)\right),
\end{aligned}
$$

The orthogonal basis $\left\{\xi_{i}\right\}$ depends of course on the specific form of the interaction, but in all the cases studied here, it satisfy the following properties: (i) The functions are either linear combinations of $\sin (s)$ or of 0 separately. There are no mixtures of sin and cos. (ii) All the functions are reduced to the expressions corresponding to the free case in the limit when the interaction parameter is sent to zero.
${ }^{1}$ A clear and interesting introduction to Fermi liquid theory can be found in A. J. Leggett, Rev. Mod. Phys. 47, 331 (1975).

${ }^{2}$ I. J. Pomeranchuk, Sov. Phys. JETP 8, 361 (1958).

${ }^{3}$ H. Yamase and W. Metzner, Phys. Rev. B 75, 155117 (2007).

${ }^{4}$ A. Neumayr and W. Metzner, Phys. Rev. B 67, 035112 (2003).

${ }^{5}$ W. Metzner, J. Reiss, and D. Rohe, Phys. Status Solidi B 243, 46 (2006).

${ }^{6}$ C. M. Varma and L. Zhu, Phys. Rev. Lett. 96, 036405 (2006); C. M. Varma, Philos. Mag. 85, 1657 (2005).

${ }^{7}$ H. Yamase and H. Kohno, J. Phys. Soc. Jpn. 69, 2151 (2000); A. Miyanaga and H. Yamase, Phys. Rev. B 73, 174513 (2006); H. Yamase and W. Metzner, ibid. 73, 214517 (2006); H. Yamase, ibid. 75, 014514 (2007).

${ }^{8}$ J. Quintanilla and A. J. Schofield, Phys. Rev. B 74, 115126 (2006).

${ }^{9}$ J. Quintanilla, C. Hooley, B. J. Powell, A. J. Shofield, and M. Haque, Physica B (Amsterdam) 403, 1279 (2008).

${ }^{10}$ C. Wu, K. Sun, E. Fradkin, and S. C. Zhang, Phys. Rev. B 75, 115103 (2007).

${ }^{11}$ J. Nilsson and A. H. Castro Neto, Phys. Rev. B 72, 195104 (2005).

${ }^{12}$ R. Gonczarek, M. Krzyzosiak, and M. Mulak, J. Phys. A 37, 4899 (2004).

${ }^{13}$ S. A. Kivelson, E. Fradkin, and V. J. Emery, Nature (London) 393, 550 (1998); V. Oganesyan, S. A. Kivelson, and E. Fradkin, Phys. Rev. B 64, 195109 (2001).

${ }^{14}$ C. J. Halboth and W. Metzner, Phys. Rev. Lett. 85, 5162 (2000);
H. Yamase and H. Kohno, J. Phys. Soc. Jpn. 69, 332 (2000).

${ }^{15}$ I. Khavkine, Chung-Hou Chung, Vadim Oganesyan, and HaeYoung Kee, Phys. Rev. B 70, 155110 (2004).

${ }^{16}$ V. Hankevych and F. Wegner, Eur. Phys. J. B 31, 333 (2003).

${ }^{17}$ A. P. Kampf and A. A. Katanin, Phys. Rev. B 67, 125104 (2003).

${ }^{18}$ Ying-Jer Kao and Hae-Young Kee, Phys. Rev. B 76, 045106 (2007).

${ }^{19}$ Christoph Puetter, Hyeonjin Doh, and Hae-Young Kee, Phys. Rev. B 76, 235112 (2007).

${ }^{20}$ S. A. Grigera et al., Science 306, 1154 (2004).

${ }^{21}$ S. A. Grigera, R. A. Borzi, A. P. Mackenzie, S. R. Julian, R. S. Perry, and Y. Maeno, Phys. Rev. B 67, 214427 (2003).

${ }^{22}$ H. Yamase and A. A. Katanin, J. Phys. Soc. Jpn. 76, 073706 (2007).

${ }^{23}$ H. Y. Kee and Y. B. Kim, Phys. Rev. B 71, 184402 (2005).

${ }^{24}$ J. M. Luttinger, Phys. Rev. 119, 1153 (1960).

${ }^{25}$ Finding $s$ analytically could be a non-trivial task. An alternative procedure to deal with Eq. (2.21) will be presented elsewhere (Ref. 29).

${ }^{26}$ P. A. Frigeri, C. Honerkamp, and T. M. Rice, Eur. Phys. J. B 28, 61 (2002).

${ }^{27}$ Y. Fuseya, H. Maebashi, and S. Yotsuhashi, J. Phys. Soc. Jpn. 69, 2158 (2000).

${ }^{28}$ For an exellent report about $d_{x^{2}-y^{2}}$ paring in cuprate superconductors see D. J. Scalapino, Phys. Rep. 250, 329 (1995).

${ }^{29}$ D. C. Cabra, C. A. Lamas, and N. Grandi (unpublished). 